\title{
TEM Analysis of Structural Transformation in Al/Ni Nanomaterials under High Energy Ion Irradiation
}

Khachatur Manukyan $^{1}$, Sergei Rouvimov ${ }^{2,3}$, Christopher E. Shuck ${ }^{4}$ and Alexander S. Mukasyan ${ }^{3,4}$

1. Department of Physics, University of Notre Dame, Notre Dame, Indiana 46556, USA

2. Department of Electrical Engineering, University of Notre Dame, Notre Dame, Indiana 46556, USA

3. Notre Dame Integrated Imaging Facility, University of Notre Dame, Notre Dame, Indiana 46556, USA

4. Department of Chemical and Bio-molecular Engineering, University of Notre Dame, Notre Dame, Indiana 46556, USA

The paper addresses the effect of irradiation by accelerated ion beam on the structural transformation of $\mathrm{Al} / \mathrm{Ni}$ multilayer nanomaterials studied by Transmission Electron Microscopy (TEM). The Al/Ni multi-layered nanomaterials are promising nanostructured energetic composite materials [1-2] that exhibit tunable ignition properties to a variety of external excitation methods including friction, shock waves, electrical sparks, and local heating. Because the ignition of such materials depends on their atomic structure and composition, irradiation may provide a novel approach for modification of the reactivity of the nanostructured energetic composite materials. Here we study the structural transformation in $\mathrm{Al} / \mathrm{Ni}$ layers under irradiation.

Magnetron sputtering and electron beam evaporation have been used to fabricate free-standing reactive multilayer nanostructured foils [3]. High energy carbon and aluminum ion beams with different charge states and intensities were used to irradiate the samples. The samples were analyzed by TEM using both high resolution TEM (HRTEM) and High Angle Annual Dark Field (HAADF) scanning TEM (STEM) modes at FEI Titan 80-300 electron microscope. The microscope was operated at 300 $\mathrm{keV}$ and equipped with an Oxford Inca EDX detector. A TEM cross-sectional sample that included the NiO-Ni interface was prepared from the top surface by Focus Ion Beam (FIB) using FEI Helios SEM/FIB dual beam equipment.

It has been demonstrated that a significant enhancement of reactivity of $\mathrm{Al} / \mathrm{Ni}$ materials after relatively short-term (40 min) high energy $\left(20 \mathrm{MeV}\right.$ ) irradiation by ${ }^{12} \mathrm{C}^{4+}$ ions (below the ignition threshold), is associated with structural transformations that lead to a decrease in the thermal selfignition temperature and ignition delay time. Indeed x-ray diffraction (Fig. 1) indicates that defect formation in the samples under irradiation leads to a decrease of the diffraction peak intensities of $\mathrm{Al}$ and $\mathrm{Ni}$ in irradiated materials as compared to the original foils. At the same time, the full width at half maximum (FWHM) of the $\mathrm{Al}$ and Ni peaks (not shown) exhibits different trends with irradiation time indicating that longer irradiation can facilitate the growth of Al crystallites while decreasing $\mathrm{Ni}$ crystallite size. This observation agrees well with TEM/STEM analysis (Figs. 1 and 2) that evidences the intermixing of $\mathrm{Al}$ and $\mathrm{Ni}$ at layer interfaces. Both high resolution TEM and electron diffraction indicate that formation of amorphous materials at the interfaces with small $(2-3 \mathrm{~nm})$ crystals of the $\mathrm{Al}_{3} \mathrm{Ni}$ intermetallic phase occur in the amorphous regions. It can be seen that the nuclei of $\mathrm{Al}_{3} \mathrm{Ni}$ crystals are distributed in the Al-rich phase close to every other $\mathrm{Al} / \mathrm{Ni}$ interface for the 40 min irradiated foil (Fig. 2). It is interesting that these nuclei line-up perpendicular to the direction of the incident beam. Such structures confirm that the beam induces solid-state diffusion of $\mathrm{Ni}$ into the $\mathrm{Al}$ layer, where nucleation of $\mathrm{Al}_{3} \mathrm{Ni}$ phase takes place.

Thus, the enhancement of multilayer energetic nanomaterial reactivity is shown to be associated with 
radiation-induced structural transformations including defect formation and intermixing of metals at the interfaces that lead to presence of amorphous layers at the interfaces with $\mathrm{Al}_{3} \mathrm{Ni}$ intermetallic nuclei.

\section{References:}

[1] Zhou, X.; et al . ACS Appl. Mater. Interfaces 2014, 6, 3058-3074

[2] Dreizin, E. L.. Prog. Energy Combust. Sci. 2009, 35, 141-167.

[3] Weihs, T.P., In Metallic Films for Electronic, Magnetic, Optical and Thermal Applications: Structure, Processing and Properties, K. Barmak and K. R. Coffey, Eds.; Woodhead Publishing, Swaston, UK, 2014, Chapter 6, 160-243
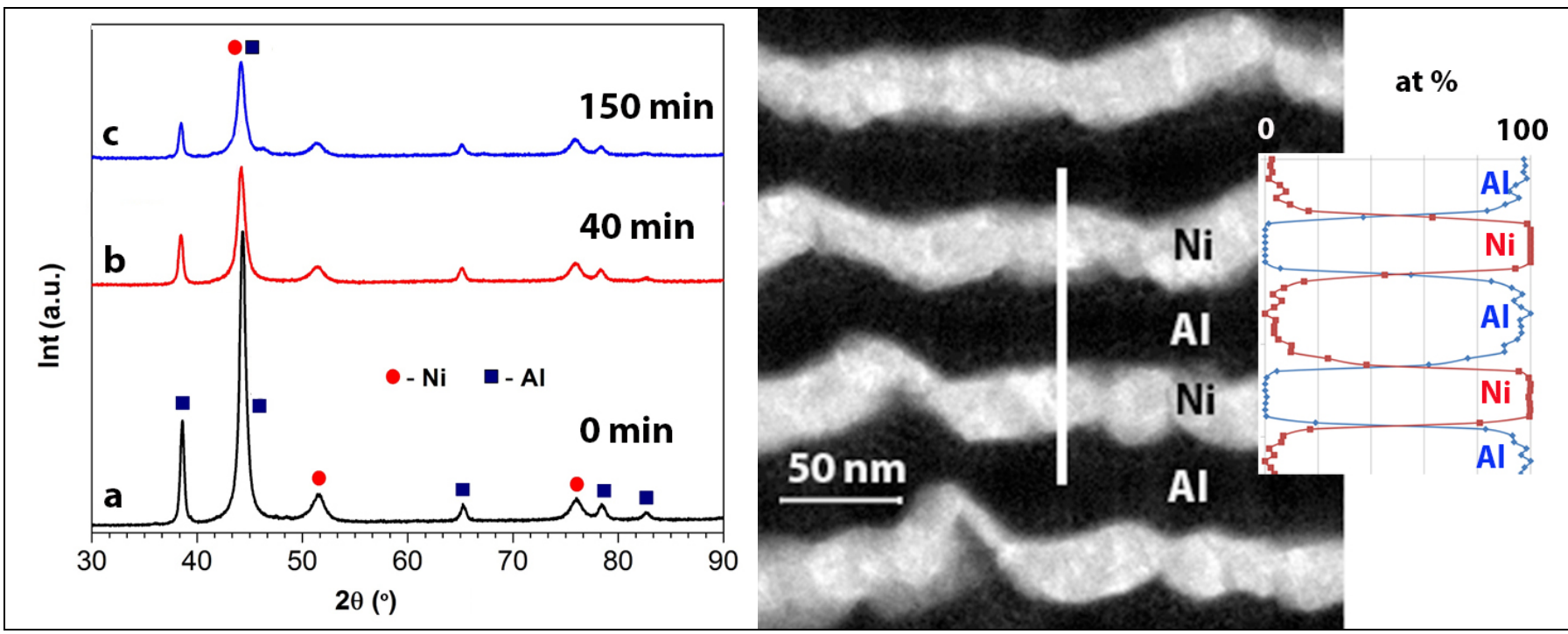

Figure 1 XRD data (left) of irradiated Ni/Al foils as compared to the original one and HAADF STEM image (right) of the $\mathrm{Ni} / \mathrm{Al}$ foil irradiated for 40 minutes. Inserted is $\mathrm{Al}$ and $\mathrm{Ni}$ distribution along the vertical line showing intermixing of $\mathrm{Al}$ and $\mathrm{Ni}$ at the interfaces.

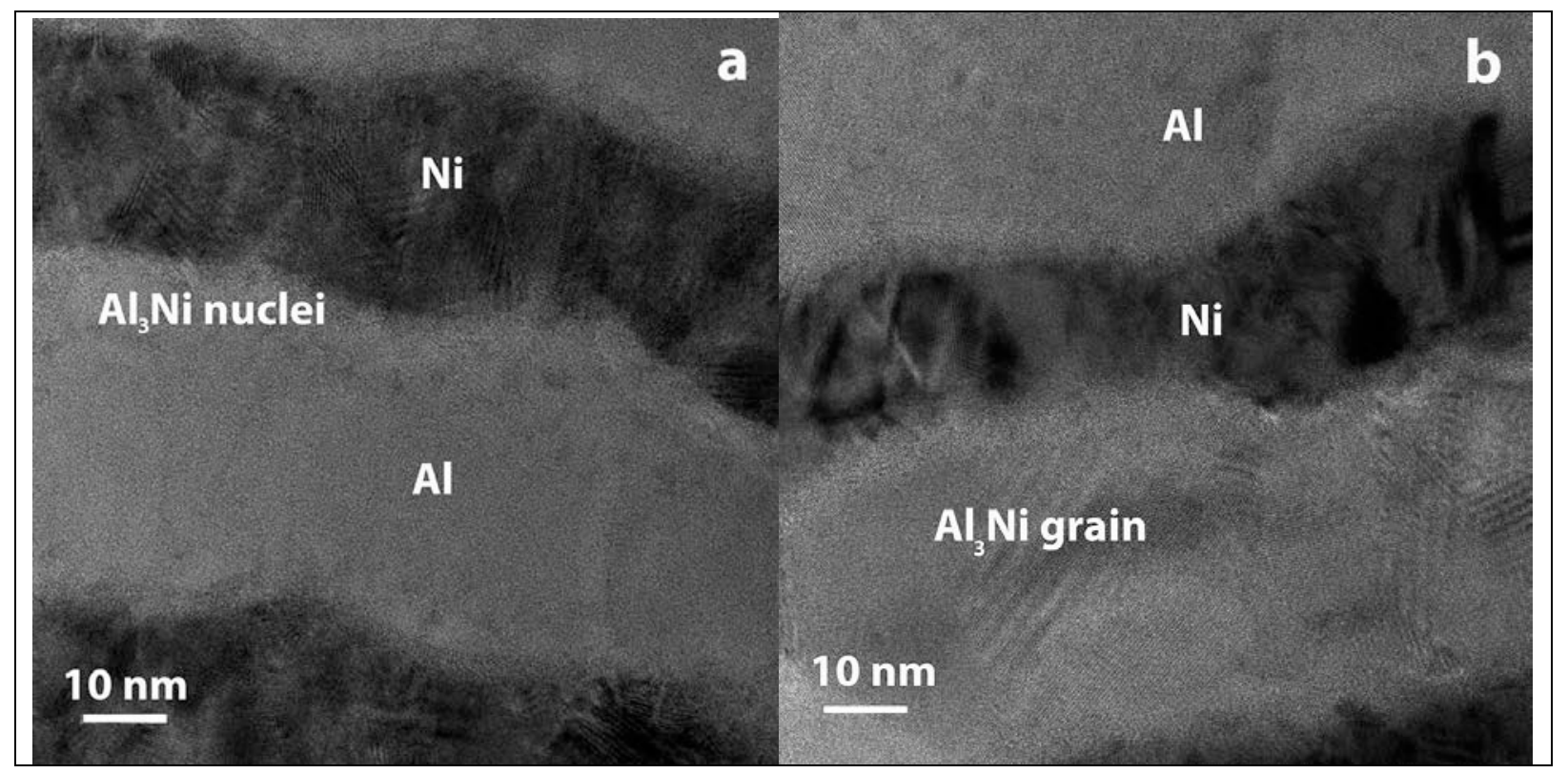

Figure 2 TEM images of Al-Ni foils irradiated for 40 (a) and 150 (b) minutes showing the Al and $\mathrm{Ni}$ intermixing and the formation of $\mathrm{Al}_{3} \mathrm{Ni}$ nuclei (a) and small $\mathrm{Al}_{3} \mathrm{Ni}$ grains (b). 